\title{
DETERMINAÇÃO DE ÁREAS DE ABRANGÊNCIA DE AGÊNCIA BANCÁRIA PELO DIAGRAMA DE VORONOI COM OBSTÁCULOS
}

Guidlines for articles formatted to publications in Bulletin of Geodetic Sciences

\author{
ANA MARIA DOS SANTOS CARNASCIALI ${ }^{1}$ \\ LUCIENE STAMATO DELAZARI ${ }^{2}$ \\ DANIEL RODRIGUES DOS SANTOS ${ }^{2}$
}

\author{
${ }^{1}$ Instituto Brasileiro de Pós-Graduação e Extensão - IBPEX \\ Rua Treze de Maio, 538 - Centro - Curitiba - PR. \\ ana.carnasciali@gmail.com \\ ${ }^{2}$ Universidade Federal do Paraná - UFPR \\ Curso de Pós-Graduação em Ciências Geodésicas - CPGCG \\ Departamento de Geomática - Curitiba - PR, Brasil \\ luciene@ufpr.br; danielsantos@ufpr.br
}

\section{RESUMO}

Os diagramas de Voronoi permitem a subdivisão das regiões em um conjunto de áreas de abrangência, a fim de estabelecer relações de proximidades. Vários estudos foram realizados para a determinação de áreas de abrangência com o uso dos diagramas de Voronoi. Entretanto, ainda é necessário aprofundar as investigações com o desenvolvimento de novas proposições, com vistas à determinação de áreas de abrangência que se aproximem da realidade topográfica das cidades. Para tal, é necessário que sejam considerados os obstáculos, ou seja, barreiras lineares e fechadas. De acordo com a literatura consultada, não existem pesquisas que tenham avançado nesse sentido, o que enfatiza a sua importância, sendo este tema desenvolvido nessa pesquisa descrita no presente artigo. Assim, foi realizada a implementação computacional dos diagramas de Voronoi ordinário e por potência (power Voronoi) com a consideração dos obstáculos, bem como realizadas análises espaciais a partir dos mesmos, estabelecendo-se relações de proximidade com os estabelecimentos dos bancos concorrentes. A partir dos resultados verificou-se que a inclusão das barreiras modificou sensivelmente as fronteiras dos diagramas de Voronoi e que, com o método proposto, evitam-se conclusões incorretas pelos usuários. Isso se explica pelo fato de que as áreas de abrangência determinadas com 
a consideração das barreiras lineares e fechadas incorporam-se à realidade topográfica das cidades.

Palavras-chave: Localização; Diagrama de Voronoi; Áreas de Abrangência; Agências Bancárias.

\section{ABSTRACT}

Voronoi diagrams allow the subdivision of a set of coverage areas in order to establish proximity relations. Several studies have been conducted to determine coverage areas by using Voronoi diagrams. However, it is still necessary to undertake further research with the development of new proposals objecting the determination of coverage areas which take into account the real topography of cities. This will require obstacles consideration, i.e., linear as well as closed barriers. According to the literature, there are no studies that have advanced in that direction, which emphasizes the importance of this research. This paper proposes to determine coverage areas by using the Voronoi diagram considering linear and closed barriers simultaneously. A computational implementation of ordinary and power Voronoi diagrams considering obstacles was performed. Spatial analyses were conducted from ordinary and power Voronoi diagrams establishing proximity relationships between competitor banks. From the results, it was found that the inclusion of barriers noticeably change the boundaries of Voronoi diagrams and that the proposed method avoids incorrect conclusions, since the coverage areas determined considering linear and closed barriers indeed approached the real topography of cities.

Keywords: Location; Voronoi Diagram; Coverage Areas; Bank Agencies.

\section{INTRODUÇÃO}

Um tópico importante no estudo da localização geográfica de um ponto, representativo de uma variável, é a determinação de sua área de abrangência ou influência. Essas áreas podem ser definidas através de estruturas geométricas denominadas diagramas de Voronoi. Os diagramas de Voronoi permitem a subdivisão das regiões em um conjunto de áreas de abrangência, a fim de estabelecer relações de proximidades, por exemplo, de uma empresa com seus concorrentes. Diversos estudos foram realizados com a utilização dos diagramas de Voronoi para a determinação de áreas de abrangência de supermercados, hospitais e escolas, o que comprova suas amplas possibilidades de aplicação (REZENDE et al., 2000; LOBO, 2003; GALVÃO et al., 2006; NOVAES et al., 2009). A determinação de áreas de abrangência com os diagramas de Voronoi ainda requer estudos que considerem os obstáculos, isto é, barreiras lineares e barreiras fechadas, concomitantemente.

Este trabalho apresenta a determinação de áreas de abrangência das agências do Banco HSBC de Curitiba (PR) pelo diagrama de Voronoi, levando em conta os obstáculos. Assim, foi desenvolvido um programa computacional para a 
determinação do diagrama de Voronoi considerando os obstáculos, bem como para a realização de análises espaciais a partir do diagrama de Voronoi ordinário e por potência sem e com a consideração das barreiras lineares e fechadas, estabelecendose relações de proximidades com os bancos concorrentes. Através dos resultados constatou-se que a incorporação das barreiras modificou consideravelmente as fronteiras dos diagramas de Voronoi e que, com o método proposto, evitam-se conclusões incorretas pelos usuários, pois as áreas de abrangência determinadas com a consideração dos obstáculos se incorporam à realidade topográfica das cidades.

\section{FUNDAMENTAÇÃO TEÓRICA}

\subsection{Diagrama de Voronoi Ordinário}

O diagrama de Voronoi para um conjunto de pontos de interesse (pontos geradores) é um conjunto de células. Cada célula está associada a um ponto gerador e contém todos os pontos mais próximos de seu ponto gerador do que de qualquer outro ponto gerador (AURENHAMMER, 1991). De acordo com Okabe, Boots e Sugihara (1992), a formulação matemática para este conceito é:

Seja $\mathrm{P}=\left\{\mathrm{p}_{1}, \mathrm{p}_{2}, \mathrm{p}_{3}, \mathrm{p}_{4}, \ldots, \mathrm{p}_{\mathrm{n}}\right\}$ um conjunto de pontos no plano euclidiano, com 2 $\leq \mathrm{n}<\infty$. Cada ponto $\mathrm{p}_{\mathrm{i}}$ tem coordenadas cartesianas $\left(\mathrm{x}_{\mathrm{i}}, \mathrm{y}_{\mathrm{i}}\right)$ e $\mathrm{p}_{\mathrm{i}} \neq \mathrm{p}_{\mathrm{j}}$ para $\mathrm{i} \neq \mathrm{j}$, i e j $\in\{1,2, \ldots, \mathrm{n}\}$. Toma-se um ponto $\mathrm{p}$ qualquer do plano Euclidiano com coordenadas $(\mathrm{x}, \mathrm{y})$. A distância euclidiana de $\mathrm{p}$ até $\mathrm{p}_{\mathrm{i}}$ é dada por:

$$
d\left(p, p_{i}\right)=\sqrt{\left(x_{i}-x\right)^{2}+\left(y_{j}-y\right)^{2}}=\left\|p-p_{i}\right\|
$$

Se $d\left(p, p_{i}\right) \leq d\left(p, p_{j}\right)$, com $j \neq i$, o ponto $p$ é designado para $p_{i}$ e a região $V\left(p_{i}\right)$, chamada região de Voronoi associada ao ponto $\mathrm{p}_{i}$ ' é definida por:

$$
V\left(p_{i}\right)=\left\{p \text { tal qued }\left(p, p_{i}\right) \leq d\left(p, p_{j}\right), \text { para } j \neq i\right\}
$$

Ao diagrama $\mathrm{V}$ gerado por $\mathrm{P}$ chama-se diagrama de Voronoi, dado por:

$$
V=\left\{V\left(p_{1}\right), V\left(p_{2}\right), \ldots, V\left(p_{n}\right)\right\}
$$

Denomina-se $\mathrm{p}_{\mathrm{i}}$ de ponto gerador e o conjunto $\mathrm{P}=\left\{\mathrm{p}_{1}, \mathrm{p}_{2}, \mathrm{p}_{3}, \mathrm{p}_{4}, \ldots, \mathrm{p}_{\mathrm{n}}\right\}$ corresponde ao conjunto gerador do diagrama de Voronoi V. No diagrama de Voronoi ordinário assume-se que os pontos geradores têm o mesmo peso e sua construção depende apenas da distância entre eles. 


\subsection{Generalizações do Diagrama de Voronoi}

A concepção do diagrama de Voronoi ponderado é análoga à do diagrama ordinário. Parte-se do conjunto de pontos geradores $\mathrm{P}=\left\{\mathrm{p}_{1}, \mathrm{p}_{2}, \mathrm{p}_{3}, \mathrm{p}_{4}, \ldots, \mathrm{p}_{\mathrm{n}}\right\} \subset$ $\mathrm{R}^{\mathrm{m}}$, com $2 \leq \mathrm{n}<\infty$ e um peso associado a cada $\mathrm{p}_{\mathrm{i}}$. Esse peso é representado por um conjunto de parâmetros $\mathrm{W}=\left\{\mathrm{w}_{1}, \mathrm{w}_{2}, \ldots, \mathrm{w}_{\mathrm{n}}\right\}$. Com esse peso é definida uma distância $\mathrm{d}_{\mathrm{w}}\left(\mathrm{p}, \mathrm{p}_{\mathrm{i}}\right)$ de $\mathrm{p}$ para $\mathrm{p}_{\mathrm{i}}$, denominada distância ponderada. A região de domínio de $\mathrm{p}_{\mathrm{i}}$ sobre $\mathrm{p}_{\mathrm{j}}$, com a distância ponderada é escrita como:

$$
\operatorname{Dom}\left(p_{i}, p_{j}\right)=\left\{\text { ptal que }_{w}\left(p, p_{i}\right) \leq d_{w}\left(p, p_{j}\right), \text { com } i \neq j\right\}
$$

e a região de Voronoi ponderada $\mathrm{V}\left(\mathrm{p}_{\mathrm{i}}\right)$ associada ao ponto $\mathrm{p}_{\mathrm{i}}$ é a interseção das regiões de domínio de $\mathrm{p}_{\mathrm{i}}$ sobre todos os $\mathrm{p}_{\mathrm{j}}$, ou seja,

$$
V\left(p_{i}\right)=\bigcap_{\forall j \neq i} \operatorname{Dom}\left(p_{i}, p_{j}\right)
$$

Existem diferentes formas de calcular a distância ponderada o que resulta em variações nas formas das regiões de domínio associadas a $\mathrm{p}_{\mathrm{i}}$. Para cada uma das diferentes formas, o diagrama recebe um nome, como por exemplo, diagrama de Voronoi por potência (power Voronoi) apresentado a seguir.

\subsection{Diagrama de Voronoi por Potência (Power Voronoi)}

Segundo Okabe, Boots e Sugihara (1992), esse diagrama é caracterizado pela distância ponderada dada por:

$$
d_{p w}\left(p, p_{i}\right)=\left\|p-p_{i}\right\|^{2}-w_{i}
$$

A região de domínio de $\mathrm{p}_{\mathrm{i}}$ sobre $\mathrm{p}$ é escrita como segue:

$$
\left.\operatorname{Dom}_{p w}\left(p_{i}, p_{j}\right)=\left\{p \text { tal que }\left\|p-p_{i}\right\|^{2}-w_{i} \leq\left\|p-p_{j}\right\|^{2}-w_{j}\right\} j \neq i\right\}
$$

Quando $\mathrm{w}_{\mathrm{i}}$ e $\mathrm{w}_{\mathrm{j}}$ são iguais a zero, a fronteira entre as duas regiões de Voronoi coincide com a mediatriz da reta que une os dois pontos geradores e o diagrama

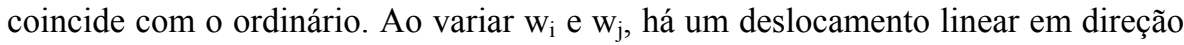
ao mais baixo valor de w (SILVA, 2004). Nesse diagrama, as regiões associadas a cada ponto gerador são sempre convexas.

\subsection{Diagrama de Voronoi com Obstáculos}

O diagrama de Voronoi é definido em função da distância euclidiana, assumindo-se, com isso, que seja sempre possível fazer uma ligação em linha reta entre quaisquer dois pontos do plano. Porém, em muitas aplicações reais isto não é 
possível. Por exemplo, uma região com parques e lagos, que não possam ser atravessados. Segundo Okabe, Boots e Sugihara (1992), se esses obstáculos se encontram entre a linha reta que une uma origem e um destino, pode-se presumir que exista uma maneira de contorná-lo. Quando isso ocorre, diz-se que o problema é com obstáculos.

Seja o conjunto de pontos geradores $\mathrm{P}=\left\{\mathrm{p}_{1}, \mathrm{p}_{2}, \mathrm{p}_{3}, \mathrm{p}_{4}, \ldots, \mathrm{p}_{\mathrm{n}}\right\} \subset \mathrm{R}^{2}$, com 2 $\leq \mathrm{n}<\infty$ e o conjunto de obstáculos $\mathrm{O}=\left\{\mathrm{o}_{1}, \mathrm{o}_{2}, \mathrm{o}_{3}, \mathrm{o}_{4}, \ldots, \mathrm{o}_{\mathrm{no}}\right\} \subset \mathrm{R}^{2}, 1 \leq \mathrm{n}_{\mathrm{o}}<$ $\infty$, que não podem ser transpostos diretamente. Assume-se que os obstáculos não podem ser sobrepostos $\left(\mathrm{o}_{\mathrm{i}} \cap \mathrm{o}_{\mathrm{j}}=\varnothing, \mathrm{i} \neq \mathrm{j}\right)$, bem como que os pontos de $\mathrm{P}$ não podem situar-se sobre os obstáculos $\left(\mathrm{p}_{\mathrm{i}} \cap \mathrm{o}_{\mathrm{j}}=\varnothing, \forall \mathrm{j}\right)$. Os obstáculos são fechados e não contêm aberturas (holes), assume-se que os obstáculos são polígonos, não necessariamente convexos (OKABE; BOOTS; SUGIHARA, 1992).

De acordo com a Figura 1 define-se a distância entre o ponto $\mathrm{p}$ e o ponto $\mathrm{p}_{1}$, pertencente ao conjunto gerador $\mathrm{P}=\left\{\mathrm{p}, \mathrm{p}_{1}, \mathrm{p}_{2}, \mathrm{p}_{3}\right\}$, pelo comprimento do menor caminho contínuo possível que os conecte sem interceptar os obstáculos pertencentes ao conjunto $\mathrm{O}=\left\{\mathrm{o}_{1}, \mathrm{o}_{2}\right\}$. Essa distância é denominada de distância do caminho mais curto (shortest-path) entre $\mathrm{p}$ e $\mathrm{p}_{1}$ e denota-se por $\mathrm{d}_{\mathrm{sp}}$.

Figura 1 - Distância do Caminho Mais Curto. Fonte: Adaptado de OKABE; BOOTS; SUGIHARA (1992).

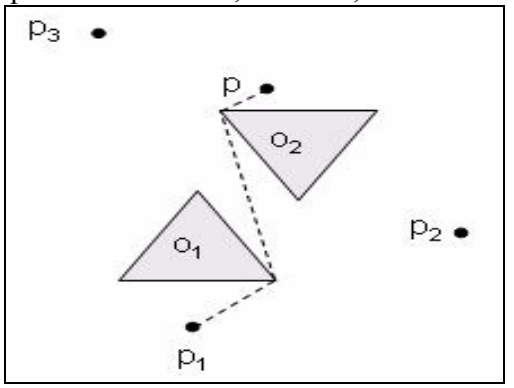

A distância do caminho mais curto é obtida com os polígonos de visibilidade e com os grafos de visibilidade. Um polígono de visibilidade, denotado por $\operatorname{VIS}\left(\mathrm{p}_{\mathrm{i}}\right)$, com respeito a $\mathrm{p}_{\mathrm{i}}$, corresponde ao conjunto de pontos que são visíveis por $\mathrm{p}_{\mathrm{i}}$. $\mathrm{VIS}\left(\mathrm{p}_{\mathrm{i}}\right)$ é definido pela expressão:

$$
\operatorname{VIS}\left(p_{i}\right)=\left\{p \text { tal que } \overline{p_{i} p} \cap\left[O_{j} \backslash \partial O_{j}\right]=\varnothing, p \in R^{2}, j \in I n_{o}\right.
$$

na qual:

$$
\overline{p_{i} p}=\text { segmento; }
$$


$\left[\mathrm{O}_{\mathrm{j}} \mid \partial \mathrm{O}_{\mathrm{j}}\right]=$ objeto restrito à fronteira, não ao interior do polígono; e $I n_{o}=$ índice de nós do grafo.

De acordo com Okabe, Boots e Sugihara (1992), o grafo de visibilidade é definido sobre um outro grafo. A Figura 2 ilustra o grafo (A) e o grafo de visibilidade (B) e assume-se que o conjunto $\mathrm{O}=\left\{\mathrm{o}_{1}, \mathrm{o}_{2}\right\}$ não é transparente, ou seja, não permite visibilidade, por exemplo, entre $\mathrm{p}_{1}$ e $\mathrm{q}_{1}$. Considera-se que o arco visível $\mathrm{L}_{\mathrm{vis}}$ é igual ao conjunto de segmentos de reta $\overline{q_{i} q_{j}}$ de tal modo que $\mathrm{q}_{\mathrm{j}}$ seja visível por $\mathrm{q}_{\mathrm{i}}$, isto é:

$$
L_{\text {vis }}=\left\{\overline{q_{i} q_{j}} \text { tal que } q_{j} \in \operatorname{VIS}\left(q_{i}\right), i<j ; i, j \in I n_{o}\right\}
$$

a expressão (9) simboliza todas as linhas representadas na Figura 2 (B), sendo o conjunto de nós $\mathrm{Q}=\left\{\mathrm{q}_{1}, \mathrm{q}_{2}, \mathrm{q}_{3}, \mathrm{q}_{4}, \mathrm{q}_{5}, \mathrm{q}_{6}, \mathrm{p}_{1}, \mathrm{p}\right\}$. Note-se que os segmentos em $\mathrm{L}_{\mathrm{vis}}$ podem se interceptar.

Figura 2 - Grafo e Grafo de Visibilidade.

Fonte: Adaptado de OKABE; BOOTS; SUGIHARA (1992).

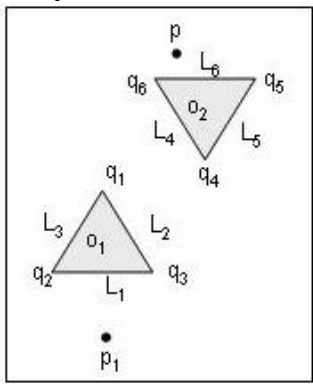

(A)

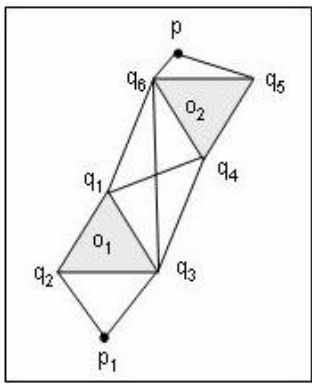

(B)

O grafo de visibilidade de $\mathrm{G}(\mathrm{Q}, \mathrm{L})$ é definido como um grafo composto de $\mathrm{Q}$ e $\mathrm{L}_{\mathrm{VIS}}$ e é denotado por $\mathrm{G}\left(\mathrm{Q}, \mathrm{L}_{\mathrm{VIS}}\right)$. Com o auxílio do grafo de visibilidade procura-se o caminho mais curto entre pontos (OKABE; BOOTS; SUGIHARA, 1992). Para facilidade de entendimento de como a distância do caminho mais curto é obtida com os polígonos de visibilidade e com os grafos de visibilidade, apresenta-se a sequência descrita em Okabe, Boots e Sugihara (1992):

- Considera-se G(Q, L), o conjunto Q dado pelos nós dos obstáculos de O e pelos pontos de $\mathrm{P}$ e L, sendo L dado pelos arcos dos obstáculos (Figura 2 (A));

- Constrói-se o grafo de visibilidade $\mathrm{G}\left(\mathrm{Q}, \mathrm{L}_{\mathrm{vis}}\right)$ (Figura 2 (B));

- Resolve-se o problema do caminho mais curto (utilizando-se um algoritmo como o de Dijkstra (CORMEN et al., 2002). 


\subsection{Método da Malha sobre uma Região}

A determinação do diagrama de Voronoi com obstáculos foi feita pelo método de uma malha definida sobre uma região. Esse método é aplicado para resolver problemas onde a resolução analítica do diagrama de Voronoi é difícil, ou seja, se torna difícil pelo fato de fazer uso de equações de retas e círculos (SILVA, 2004).

Esse método foi idealizado por Silva (2004). Segundo o autor, o procedimento para determinar a divisão do diagrama de Voronoi baseia-se em verificar de qual ponto gerador cada nó da malha está mais próximo. Convenciona-se, por exemplo, o vértice superior esquerdo de cada elemento que forma a malha como sua identificação. Assim, se esse ponto está mais próximo do ponto gerador $i$, todos os pontos desse elemento da malha também estão. Para efeito de ilustração, na Figura 3 a regiões 1, 2 e 3 correspondem aos pontos geradores 1,2 e 3 , respectivamente.

Figura 3 - Voronoi Sobre a Malha.

Fonte: Adaptado de SILVA (2004).

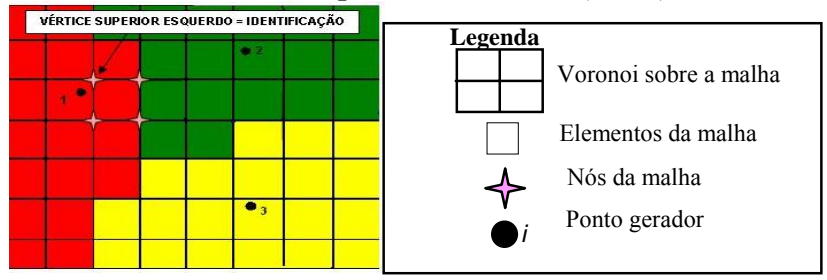

\section{MATERIAIS}

Foi utilizada a base cartográfica digital de Curitiba (PR), adquirida junto ao Instituto de Pesquisa e Planejamento Urbano de Curitiba (IPPUC), edição 2005. Os endereços das agências do HSBC foram fornecidos pelo Departamento de Administração de Imóveis (DAI) referentes a maio/2009. Os endereços das agências dos bancos concorrentes foram obtidos junto ao Banco Central do Brasil (BC) através da Divisão de Gestão de Sistemas de Informações Cadastrais, Contábeis e Econômico-Financeiras (DINFO) referentes a maio/2009.

Para a determinação dos diagramas de Voronoi ordinário e por potência, foi utilizado o programa desenvolvido neste trabalho denominado Diagrama de Voronoi com Obstáculos (DVO). O mesmo foi desenvolvido em Visual Basic 6.0.

Para a tabulação dos dados foi utilizado o software Microsoft Excel (2003). Na geocodificação das agências HSBC e agências dos bancos concorrentes, foi utilizado o software ArcGIS 9.3 (ESRI). Para a extração dos fatores, que descrevem os relacionamentos entre as variáveis, em termos de poucas variáveis fundamentais, utilizou-se o software Statgraphics Centurion XV. Todos os softwares anteriormente citados estão disponíveis na Universidade Federal do Paraná (UFPR). Foi utilizado um computador Pentium core 2 duo T8400, 4 Gb de RAM, Windows Vista 64 bits. 


\section{MÉTODOS}

A seguir serão apresentados os métodos empregados para a realização do trabalho, que engloba as barreiras lineares e fechadas, a geocodificação, a seleção das variáveis que caracterizam os pontos geradores, a sua ponderação e a implementação do diagrama de Voronoi com obstáculos.

\subsection{Barreiras Lineares e Fechadas}

Para o município de Curitiba (PR) identificaram-se como barreiras lineares as rodovias e os rios. Como barreiras fechadas, os cemitérios, clubes/associações recreativas, condomínios/loteamentos fechados, glebas/terrenos vagos, instalações comerciais (shoppings e atacadistas), instalações de transportes (aeroportos, terminais rodoviários e ferroviários), instalações educacionais/culturais (campi universitários, escolas), instalações esportivas (estádios de futebol), instalações hospitalares, instalações industriais, instalações militares (quartéis), bosques, parques, Jardim Botânico Francisca Maria Garfunkel Rischbieter, Passeio Público, Setor Esportivo Peladeiros, lagos e ferrovias (cercada). Com exceção dos bosques, parques, Jardim Botânico, Passeio Público, Setor Esportivo Peladeiros e os lagos, as demais barreiras fechadas foram identificadas e delimitadas.

As barreiras podem ser transpostas apenas em pontos específicos, que não correspondem, dessa maneira, à totalidade de suas extensões. Elas podem ser transpostas por viadutos, trincheiras, passarelas e pontes, que foram denominados de pontos de passagem.

\subsection{Geocodificação}

Como as 29 agências do HSBC e as 236 agências dos bancos concorrentes: ABN AMRO Real S/A, Bradesco S/A, Citibank S/A, Banco do Brasil S/A, Itaú S/A, Santander S/A, Bankboston S/A, Caixa Econômica Federal e Unibanco S/A, não fazem parte da base cartográfica digital do IPPUC, foi feita a geocodificação a partir dos endereços, no software ArcGIS 9.3 (ESRI), utilizando-se a função geocode. No processo de geocodificação, foi realizada a interpolação ao longo dos trechos de logradouro, observando-se as faixas de numeração predial associadas a cada trecho, sendo mapeado cada registro das tabelas. A base cartográfica contém um campo chamado TRECHO_RUA que foi utilizado para a geocodificação. Nele está incluída além da numeração predial inicial e final de cada trecho, a denominação e codificação oficiais das ruas.

\subsection{Variáveis que Caracterizam os Pontos Geradores}

Os pontos geradores foram as agências do HSBC. Nas áreas de abrangência determinadas pelo diagrama de Voronoi ordinário, os pontos geradores possuem o mesmo peso e sua construção depende apenas da distância entre eles. Nos diagramas de Voronoi ponderados, os pontos geradores podem assumir pesos 
diferenciados. Assim, seguindo esse conceito, foi realizada a determinação de variáveis que os caracterizem. Conjuntamente, com os especialistas do DAI e especialistas dos Bancos Bradesco e do extinto Bamerindus, foram selecionadas as variáveis: categoria, vigência, horário, área, caixas, auto-atendimento, publicidade, vagas de estacionamento, sanitários e acesso a portador de deficiência física/mobilidade reduzida. Tais variáveis foram selecionadas pelo fato de permitir a comparação das agências bancárias em termos de serviços, facilidades para os clientes e publicidade/apoio.

\subsection{Ponderação dos Pontos Geradores}

Para descrever os relacionamentos entre as variáveis citadas no item 4.3, em termos de poucas variáveis fundamentais, denominadas fatores, foi necessária a realização de uma análise fatorial. Para a extração dos fatores, foi aplicado o método das componentes principais (JOHNSON; WICHERN, 1988) e utilizou-se o critério de Kaiser, que seleciona aqueles que apresentam autovalor $(\lambda)$ maior do que 1.0 (FIELD, 2005). Analisados os valores referentes à percentagem acumulada, verificou-se que três fatores extraídos explicam $75,028 \%$ da variabilidade dos dados originais. Portanto, foi considerado esse número de fatores. Para interpretar os fatores, os mesmos foram rotacionados pelo método Varimax. A partir dos fatores rotacionados foram obtidos os escores brutos para cada ponto gerador, que correspondem aos pesos. Para a sua determinação utilizou-se a equação:

$$
E s C_{b j}=\frac{\sum_{i=1}^{m} \lambda_{i j} f_{i j}}{\sum_{i=1}^{m} \lambda_{i j}} \quad j=1.2 \ldots . . m
$$

no qual $\lambda_{i j}=$ autovalor e $f_{i j}=$ escore fatorial.

Os pesos resultaram: Ag. Avenida/Matriz, 1,984, Ag. Com. Araújo, 1,242, Ag. Centro Cívico, 0,896, Ag. Água Verde, 0,841, Ag. João Negrão 0,493, Ag. Juvevê, 0,453, Ag. Portão, 0,375, Ag. Bacacheri, 0,044, Ag. Mercês, 0,011, Ag. Cidade Industrial, 0,004, Ag. Alto da XV, 0,004, Ag. Parolin, -0,087, Ag. Mal Deodoro, 0,090, Ag. Passarela, -0,158, Ag. Mal Floriano, -0,164, Ag. Brasílio Itiberê, -0,197, Ag. Pinheirinho, -0,226, Ag. Cristo Rei, -0,240, Ag. Ahú, -0,265, Ag. Salgado Filho, -0,283, Ag. Santa Felicidade, -0,399, Ag. Novo Mundo, -0,449, Ag. Av. Brasília, -0,456, Ag. Champagnat, -0,475, Ag. Jardim Social, -0,501, Ag. Hauer, 0,502, Ag. Seminário, -0,515, Ag. Orleans, -0,518, Ag. Ceasa, -0,824. Esses pesos foram utilizados na determinação dos diagramas de Voronoi por potência sem e com a consideração das barreiras. No diagrama de Voronoi por potência é possível perceber o deslocamento linear das fronteiras entre as regiões na direção do ponto gerador associado ao peso de valor mais baixo (Quadro 1 e Figura 9). Quando o peso é menor que zero ocorre uma diminuição da área, quando é maior que zero a área aumenta. 


\subsection{Implementação do Diagrama de Voronoi com Obstáculos (Dvo)}

Neste trabalho o método da malha citado no item 2.5, foi modificado para resolver problemas com obstáculos, não apenas lineares, mas também fechados. Para as barreiras fechadas foi aplicado o conceito de envoltório convexo. Assim, utilizou-se o algoritmo QuickHull que calcula o fecho convexo de um conjunto de pontos no plano (PREPARATA; SHAMOS, 1985). Como as barreiras fechadas não poderiam ser transpostas, foram desconsideradas as diagonais internas do envoltório convexo que as circula, pois essas diagonais poderiam resultar em uma distância de caminho mais curto que não corresponde a uma opção de transposição.

Para a determinação da distância do caminho mais curto, foram utilizados os conceitos de visibilidade entre dois pontos e grafo de visibilidade, descritos no item 2.4. A partir da determinação das distâncias dos caminhos mais curtos foi efetuada a divisão do Voronoi ordinário. Para a determinação do diagrama de Voronoi com obstáculos, o método empregado foi o mesmo utilizado para efetuar a divisão do diagrama de Voronoi ordinário, trocando-se a distância euclidiana pela distância do caminho mais curto em um grafo. Maiores detalhes e resultados práticos foram apresentados nos experimentos.

\section{EXPERIMENTOS}

Para a definição da resolução da malha foi gerado o diagrama de Voronoi ordinário com o sioftware ArcGIS e com o programa DVO, de modo a possibilitar uma comparação entre as áreas de abrangência resultantes. O software ArcGIS apresenta a função Create Thiessen Polygons (CTP) que permite traçar apenas esse tipo de diagrama, sem considerar as barreiras lineares ou fechadas. Ao traçar o diagrama a função CTP gera automaticamente um retângulo. Como o retângulo gerado interrompeu as áreas de abrangência das agências 2, 6, 8, 11, 13, 15, 22, 24, 27 e 28 , que fazem contato com a sua borda, foram calculadas as áreas das agências $1,3,4,5,7,9,10,12,14,16,17,18,19,20,21,23,25,26$ e 29 , totalizando 19 agências.

A partir do programa DVO, foram testadas divisões da malha com elementos quadrados de lados $25 \mathrm{~m}, 50 \mathrm{~m}, 100 \mathrm{~m}$ e $200 \mathrm{~m}$, para determinar com que valor trabalhar. Essas medidas foram selecionadas considerando-se a menor distância entre duas agências, que no caso correspondem às agências Avenida/Matriz e Mal. Deodoro, cuja distância linear resultou em $458 \mathrm{~m}$. Partindo-se do diagrama de Voronoi ordinário, definiram-se as linhas de fronteira entre dois pontos a partir da mediatriz que os une. Assim, a mesma estaria a $229 \mathrm{~m}$, o que levou a escolha de elementos quadrados de lados iguais ou inferiores a $200 \mathrm{~m}$. Foram calculadas as áreas das mesmas 19 agências anteriores, referentes aos elementos quadrados de lados $25 \mathrm{~m}, 50 \mathrm{~m}, 100 \mathrm{~m}$ e $200 \mathrm{~m}$.

Para possibilitar a comparação entre as áreas de abrangência determinadas com o ArcGIS e o DVO foi calculada a diferença percentual, resultando como 
máximas diferenças do DVO em relação ao ArcGIS para elementos quadrados de $25 \mathrm{~m}, 50 \mathrm{~m}, 100 \mathrm{~m}$ e $200 \mathrm{~m}, 0,38 \%, 0,57 \%, 2,63 \%$ e $5,94 \%$, respectivamente, e como médias das diferenças $0,08 \%, 0,17 \%, 0,83 \%$ e $1,72 \%$. Foram selecionados os elementos quadrados de $50 \mathrm{~m}$ para compor a malha. Esta escolha foi baseada em:

a) nenhuma diferença percentual da malha de $50 \mathrm{~m}$ resultou igual ou superior a $1 \%$, quando comparadas com as diferenças percentuais das malhas de $100 \mathrm{~m}$ e 200 $\mathrm{m} ;$

b) a quadra corresponde ao menor elemento da base cartográfica a ser considerado, cuja menor extensão linear é $60 \mathrm{~m}$. A resolução da malha igual a $50 \mathrm{~m}$ fica dentro desse limite. Para uma resolução de $25 \mathrm{~m}$ a mesma extrapola os $60 \mathrm{~m}$ da quadra, o que não justifica a sua escolha, pois a mesma equivale à dimensão linear de um lote;

c) a quantidade de divisões da malha para elementos quadrados de lados $50 \mathrm{~m}$, resultou em $\mathrm{X}=420$ e $\mathrm{Y}=680$, totalizando 285.600 elementos que compõem a malha, ou seja, 285.600 cálculos de distância. Se fossem considerados elementos quadrados de lados $25 \mathrm{~m}(\mathrm{X}=840$ e $\mathrm{Y}=1360)$ os elementos que compõem a malha totalizariam 1.142.400, ou seja, quatro vezes superior ao da malha de $50 \mathrm{~m}$.

\section{ANÁLISE DOS RESULTADOS}

A fim de estabelecer as relações de proximidade das agências HSBC com as agências dos bancos concorrentes, foram determinadas as áreas de abrangência das 29 agências do HSBC pelos diagramas Voronoi Ordinário Sem Barreiras (VOSB), Voronoi Ordinário Com Barreiras (VOCB), Voronoi Power Sem Barreiras (VPSB) e Voronoi Power Com Barreiras (VPCB), a partir do programa DVO.

\subsection{Vorornoi Ordinário sem e com Barreiras}

A Figura 4 ilustra a área de abrangência da agência HSBC 9, com as agências dos bancos concorrentes determinadas com os diagramas VOSB (A) e VOCB (B), respectivamente.

As seis agências dos bancos concorrentes que apresentaram mudanças de áreas de abrangência com os diagramas VOSB e VOCB, foram: Caixa Economica Federal da Av. Candido de Abreu, 127, Banco Itaú S.A. da Av. Desembargador Hugo Simas, 526 e R. Izaac Ferreira da Cruz, 113, Banco Bradesco S.A. da R.Gra Nicco, 3173, Banco do Brasil S.A. da R. Francisco Derosso, 4006 e R. Engenheiro Costa Barros, 1551(Figura 4). 
Figura 4 - Áreas de Abrangência Determinadas com os Diagramas VOSB e VOCB e Agências dos Bancos Concorrentes. Fonte: CARNASCIALI, 2010.

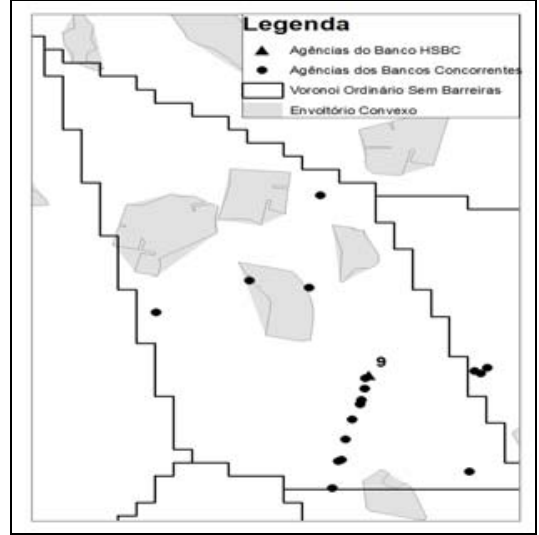

(A)

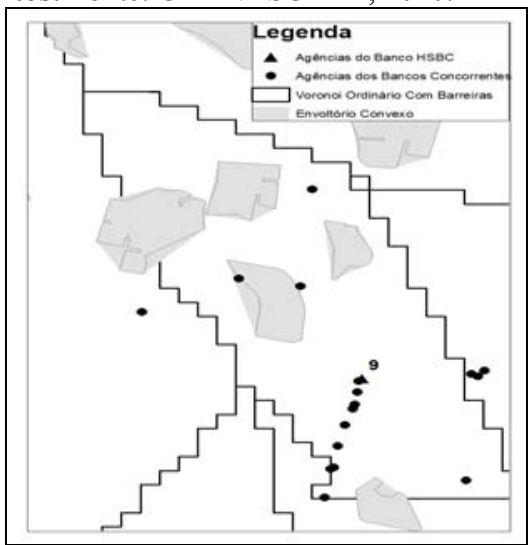

(B)

A fim de exemplificar a mudança de áreas de abrangência, considerou-se a área de abrangência da agência do Banco Bradesco S/A, da Rua Gra Nicco, 113, que de acordo com o VOSB, ficou localizada na área de abrangência da agência HSBC 22 (Figura 5 (A)) e pelo VOCB ficou localizada na área de abrangência da agência HSBC 29 (Figura 5 (B)).

Pode-se observar que a mudança de área de abrangência de ambas as agências foi devida à inclusão das barreiras (VOCB), no caso correspondentes à barreira linear referente à rodovia e as barreiras fechadas correspondentes às instalações industriais e condomínios/loteamentos fechados.

Figura 5 - Localização da Agência Bradesco da Rua Gra Nicco. Fonte: CARNASCIALI, 2010.
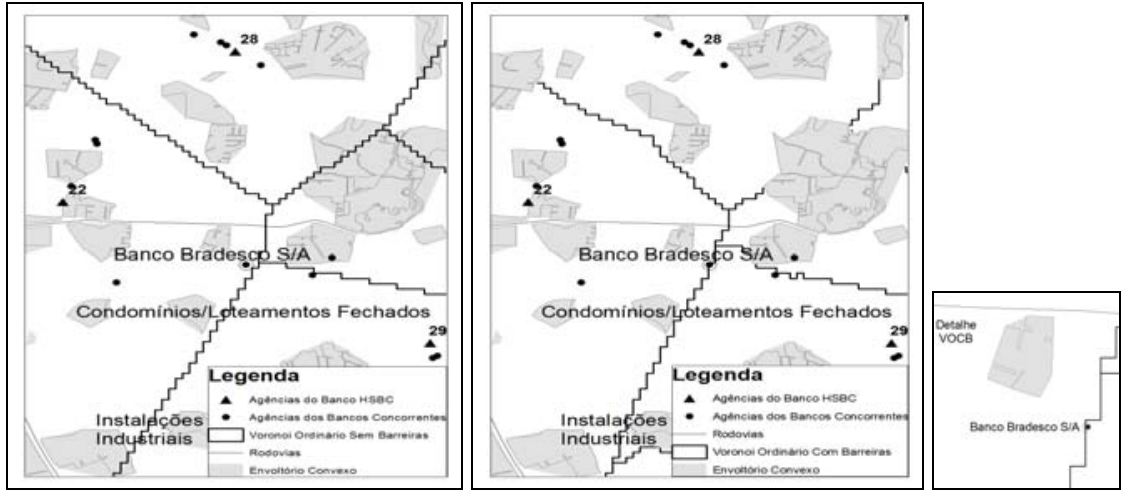

Bol. Ciênc. Geod., sec. Artigos, Curitiba, v. 17, nº 1, p.200-217, abr-jun, 2011. 

(A) VOSB
(B) VOCB

\subsection{Vorornoi por Potência sem e com Barreiras}

A Figura 6 ilustra a área de abrangência da agência HSBC 9, com as agências dos bancos concorrentes determinadas com os diagramas VPSB (A) e VPCB (B), respectivamente.

Figura 6 - Áreas de Abrangência Determinadas com os Diagrama VPSB e VPCB Agências dos Bancos Concorrentes. Fonte: CARNASCIALI, 2010.

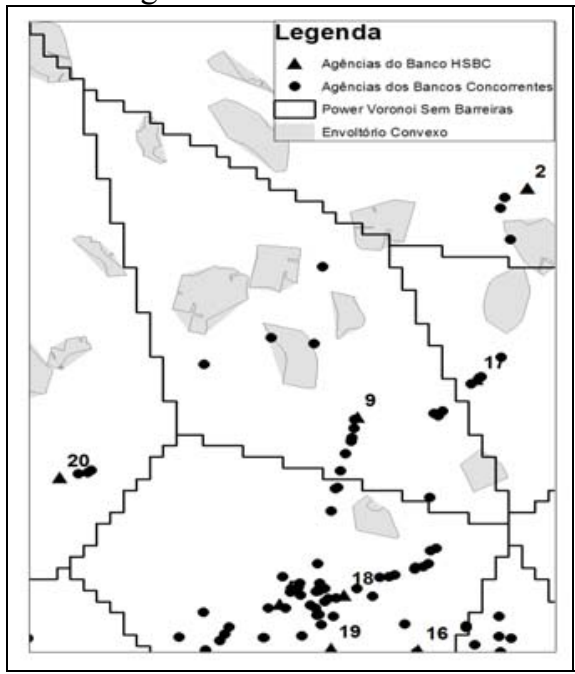

(A)

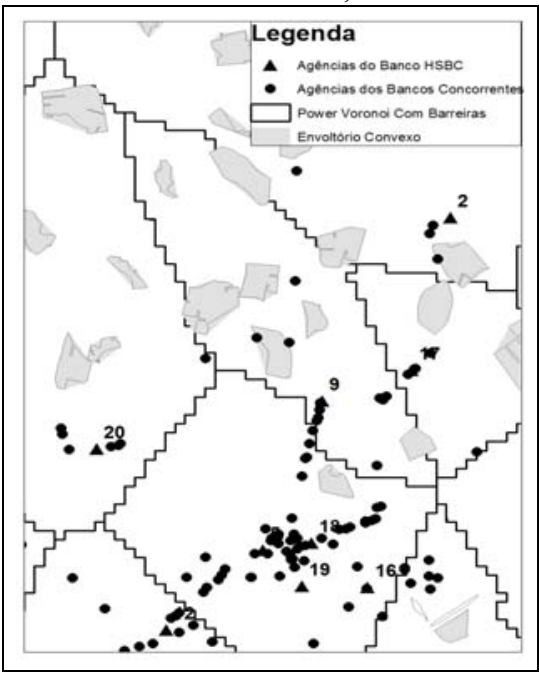

(B)

As sete agências dos bancos concorrentes que apresentaram mudanças de áreas de abrangência com os diagramas VPSB e VPCB, foram: Bankboston S.A. da Av. Cândido de Abreu, 285, Banco Bradesco S.A. da Av. Cândido de Abreu, 427, Banco Itaú S.A. da Av. Desembargador Hugo Simas, 526, Banco Bradesco S.A. da R.Gra Nicco, 113, Banco Itaú S.A. da R. Izaac Ferreira da Cruz, 3173, Banco do Brasil S.A. da R. Francisco Derosso, 4006 e R. Engenheiro Costa Barros, 1551.

De acordo com a Figura 7, ao se comparar as áreas de abrangência entre os diagramas VPSB e VPCB, é possível perceber uma sobreposição entre as fronteiras de ambos os diagramas. Isso ocorre porque ambos os diagramas correspondem ao diagrama de Voronoi por potência. As diferenças das fronteiras se evidenciam pela inclusão das barreiras lineares e fechadas. 
Figura 7 - Comparativo das Áreas de Abrangência pelos Diagramas VPSB E VPCB. Fonte: CARNASCIALI, 2010.
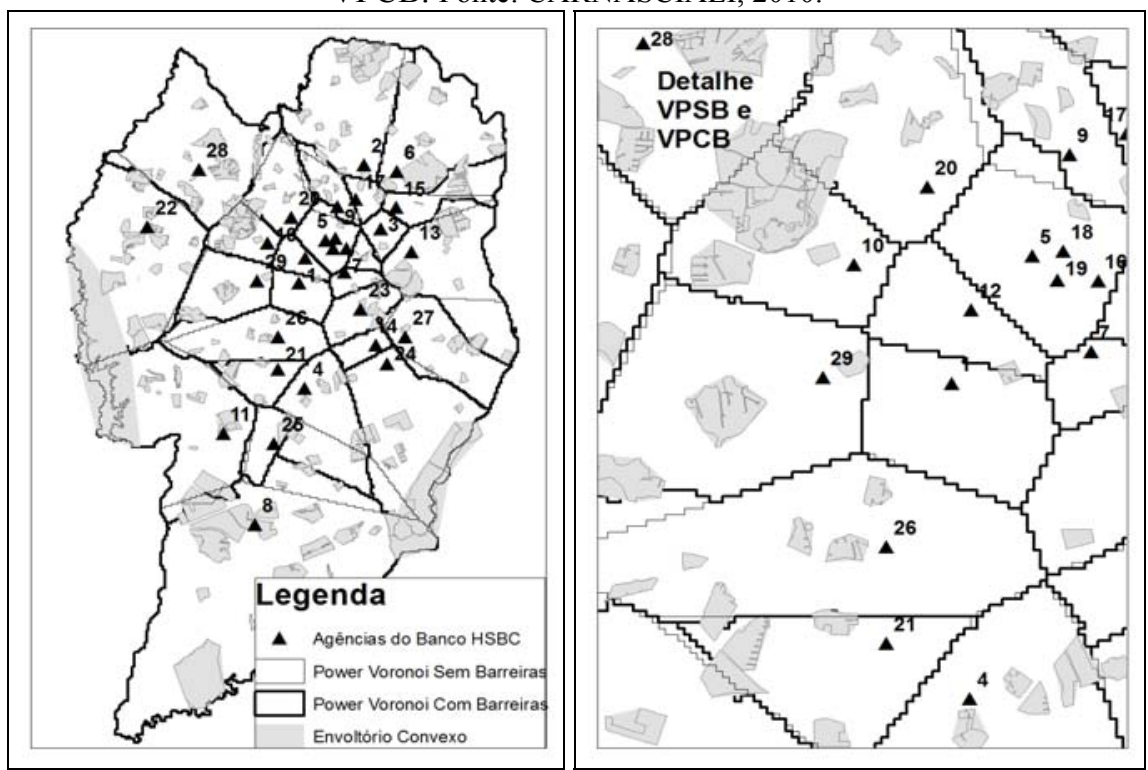

\subsection{Vorornoi Ordinário sem Barreiras e por Potência com Barreiras}

De acordo com a Figura 8, ao se comparar as áreas de abrangência entre os diagramas VOSB e VPCB, é possível perceber que as fronteiras de ambos os diagramas não se sobrepõem. Isso ocorre devido à inclusão das barreiras lineares e fechadas, bem como pelos pesos associados aos pontos geradores do diagrama de Voronoi por potência.

Para subsidiar o entendimento da não sobreposição das fronteiras entre os diagramas VOSB e VPCB, devido aos pesos associados aos pontos geradores, foi elaborado o Quadro 1 correspondente à Agência Hauer e as agências HSBC confrontantes. A Figura 9 ilustra os diagramas VOSB e VPCB para a Agência Hauer e as agências HSBC confrontantes. Para o diagrama de Voronoi ordinário, os pesos são iguais a zero e a fronteira entre duas regiões de Voronoi coincide com a mediatriz da reta que une os dois pontos geradores. Para o diagrama de Voronoi por potência é possível perceber o deslocamento linear da fronteira entre as regiões $14 \mathrm{e}$ 23 , na direção do ponto gerador 14 , pelo fato do mesmo estar associado ao peso de valor mais baixo. O mesmo acontece entre as regiões 14 e 26, 14 e 4, 14 e 24,14 e 27 , há um deslocamento linear da fronteira na direção do ponto gerador 14 , pelo fato do mesmo estar associado ao peso de valor mais baixo. 
Figura 8 - Comparativo das Áreas de Abrangência com os Diagramas VOSB e VPCB. Fonte: CARNASCIALI, 2010.
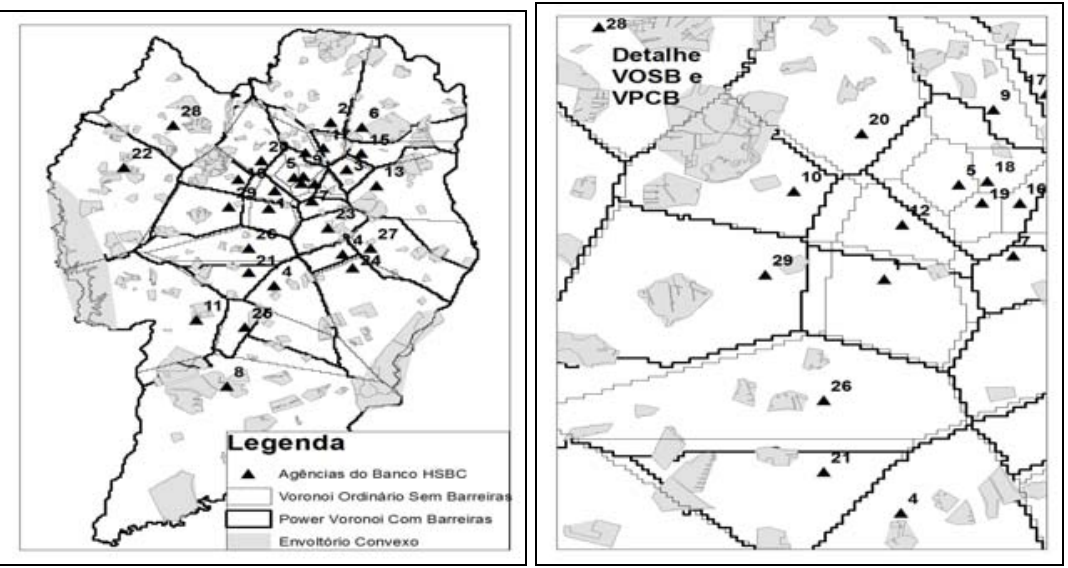

Quadro 1 - Deslocamento Linear das Fronteiras - Agência Hauer.

Fonte: CARNASCIALI, 2010

\begin{tabular}{|c|c|c|c|c|c|}
\hline \multicolumn{2}{|c|}{$\begin{array}{c}\text { Pontos Geradores } \\
\text { Agência HSBC }\end{array}$} & $\begin{array}{c}\text { Peso } \\
\text { Ag.HSBC }\end{array}$ & $\begin{array}{c}\text { Agência } \\
\text { HSBC } \\
\text { Confrontante }\end{array}$ & $\begin{array}{c}\text { Peso } \\
\text { Agência HSBC } \\
\text { Confrontante }\end{array}$ & $\begin{array}{c}\text { Deslocamen } \\
\text { to } \\
\text { linear } \\
\rightarrow \text { nara }\end{array}$ \\
\hline \multirow{5}{*}{14} & \multirow{5}{*}{$\begin{array}{l}\text { Agência } \\
\text { Hauer }\end{array}$} & \multirow{5}{*}{$-0,50245$} & 23 & $-0,08681$ & $23 \rightarrow 14$ \\
\hline & & & 26 & 0,375492 & $26 \rightarrow 14$ \\
\hline & & & 4 & $-0,45578$ & $4 \rightarrow 14$ \\
\hline & & & 24 & $-0,15758$ & $24 \rightarrow 14$ \\
\hline & & & 27 & $-0,28301$ & $27 \rightarrow 14$ \\
\hline
\end{tabular}

Figura 9 - Comparativo das Áreas de Abrangência com os Diagramas VOSB e VPCB para a Agência Hauer. Fonte: CARNASCIALI, 2010.

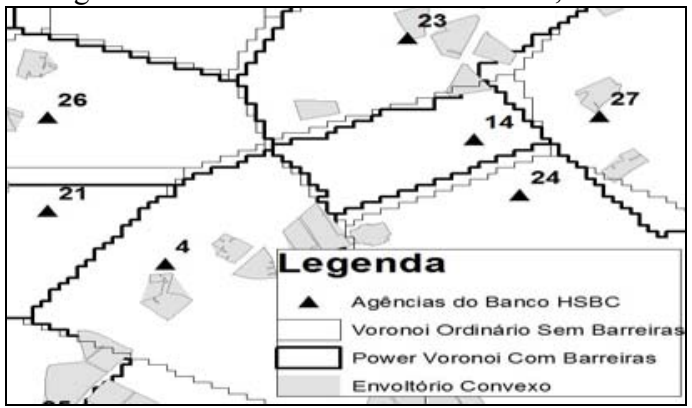

Bol. Ciênc. Geod., sec. Artigos, Curitiba, v. 17, nº 1, p.200-217, abr-jun, 2011. 


\section{CONCLUSÕES E RECOMENDAÇÕES}

Foi realizada a implementação computacional dos diagramas de Voronoi ordinário e por potência considerando as barreiras lineares e fechadas. O programa desenvolvido foi denominado Diagrama de Voronoi com Obstáculos (DVO). O DVO mostrou potencialidade para tratar adequadamente os obstáculos, que corresponderam a 192 barreiras fechadas e a 26 barreiras lineares. A fase mais crítica no tempo de processamento foi na determinação do caminho mais curto pelo algoritmo de Dijkstra, porque cada ponto da malha teve que buscar todos os pontos geradores, o que resultou em 285.600 cálculos de distância. O tempo de processamento, desde a leitura dos dados de entrada até o cálculo do diagrama, foi em torno de 14 horas. Reduzir o tempo de processamento não foi objetivo neste trabalho, pois o método proposto visa auxiliar a tomada de decisão, e não exige resposta em tempo real.

Como dificuldades de implementação pode-se destacar o número de segmentos que compõem as barreiras. Em Silva (2004), foram consideradas somente barreiras lineares correspondentes a dois rios. Os mesmos totalizaram em torno de 50 segmentos. Neste trabalho, para as barreiras lineares e fechadas, o número de segmentos foi em torno de 6.000. De acordo com a complexidade do algoritmo Dijkstra $\left(\mathrm{O}\left(\mathrm{n}^{2}\right)\right)$, considerando-se o número de segmentos usados neste trabalho pelo número de segmentos de Silva (2004), ou seja, 6.000 divididos por 50, que resultam 120, para achar um caminho mais curto, o custo equivale a $120^{2}$, isto é, 14.400 vezes maior. Partindo-se dos 285.600 cálculos de distância efetuados, multiplicados por 14.400, resultam em 4.112.640.000 vezes mais custoso se comparados a Silva (2004). O cálculo do grafo de visibilidade pode ser apontado como outra dificuldade devido ao número de segmentos das barreiras.

A inclusão tanto das barreiras fechadas como as lineares, foi importante para as análises, porque as áreas de abrangência com a consideração dos obstáculos, ficam mais próximas da realidade topográfica, como pode ser constatado pelas análises espaciais. A vantagem do método proposto quando comparado aos estudos anteriores está relacionada à consideração de barreiras reais, que muitas vezes são fechadas. Entretanto, problemas maiores que o deste trabalho, ainda demandam estudos devido à complexidade computacional na implementação das barreiras fechadas. A determinação de áreas de abrangência pode ser generalizada para qualquer ponto de interesse, ou seja, independe do domínio da aplicação, o que muda são as variáveis que caracterizam os pontos geradores.

Quanto mais próximas as áreas de abrangência estiverem da realidade topográfica das cidades, melhores serão as respostas alcançadas através das análises espaciais. Dessa forma, evitam-se conclusões incorretas pelos usuários, auxiliandoos nas tomadas de decisões com respeito à ampliação ou redução da rede de atendimento, na escolha da melhor localização de um novo ponto ou de um ponto já existente, bem como na prospecção de novos clientes. 
Como recomendações para a continuidade desta pesquisa destacam-se: a utilização dos diagramas de Voronoi gerados computacionalmente para a determinação de áreas de abrangência de pontos, combinados com indicadores socioeconômicos, através de um Sistema de Informações Geográficas (SIG), o que amplia as possibilidades de análises espaciais; a geocodificação de clientes pessoa física e jurídica, que uma vez representados nas áreas de abrangência das agências, possibilitam análises de prospecção de novos clientes.

\section{AGRADECIMENTOS}

À Coordenação de Aperfeiçoamento de Pessoal de Nível Superior (CAPES) pela concessão da bolsa. Ao HSBC Bank Brasil S/A - Departamento de Administração de Imóveis pela colaboração no desenvolvimento do trabalho.

\section{REFERÊNCIAS BIBLIOGRÁFICAS}

AURENHAMMER, F. Voronoi diagrams: a survey of a fundamental geometric data structure. ACM Computing Surveys, v. 23, n. 3, p. 345-405, 1991.

CARNASCIALI, A. M. S. Determinação de áreas de abrangência de pontos pelo diagrama de Voronoi com obstáculos. 156 f. Tese (Doutorado em Ciências Geodésicas) - Setor de Ciências da Terra, Universidade Federal do Paraná, Curitiba, 2010.

CORMEN, T. H. et al. Algoritmos: teoria e prática. Tradução da segunda edição. Rio de Janeiro: Campos, 2002. 916 p.

Curitiba. Instituto de Pesquisa e Planejamento Urbano de Curitiba - IPPUC. Mapa Digital de Curitiba. Curitiba, 2005.

EXCEL. Apostila do excel: básico intermediário e avançado. Sergio Boudart. 2003. Disponível em: $<$ http://www.apostilando.com> Acesso em: 22/09/2008.

FIELD, A. Discovering statistics using SPSS. Sage Publications Ltd: London, 2005. $779 \mathrm{p}$.

GALVÃO, L. et al. A multiplicatively-weighted Voronoi diagram approach to logistics districting. Computers and Operations Research. n. 33. p. 93-114. 2006.

JOHNSON, R. A.; WICHERN, D. W. Applied multivariate statistical analysis. 2 ed. New Jersey: Prentice-Hall, 1988. 607 p.

LOBO, D. S. Dimensionamento e otimização locacional de unidades de educação infantil. 155 f. Tese (Doutorado em Engenharia da Produção) Departamento de Transportes e Logística, Universidade Federal de Santa Catarina, Florianópolis, 2003. Disponível em: <http://www.geoinfo.info $>$ Acesso em: 18/09/2007.

NOVAES, A. G. N. et al. Solving continuous location-districting problems with Voronoi diagrams. Computers and Operations Research, n. 36, p. 40-59, 2009. 
OKABE, A.; BOOTS, B.; SUGIHARA, K. Spatial tessellations: concepts and applications of Voronoi diagrams. Chichester: John Wiley, 1992. 532 p.

PREPARATA, F. P.; SHAMOS, M. I. Computational geometry: an introduction. New York: Springer-Verlag, 1985. 398 p.

REZENDE, F. et al. Diagramas de Voronoi para a definição de áreas de abrangência de hospitais públicos no Município do Rio de Janeiro. Cadernos de Saúde Pública. Rio de Janeiro, v. 16, n. 2, p. 109-118, 2000.

SILVA, A. C. L. Estratégia para divisão de áreas de estudo em problemas logísticos: uso do diagrama de Voronoi com obstáculos. $131 \mathrm{f}$. Tese (Doutorado em Engenharia de Produção) - Departamento de Transportes e Logística, Universidade Federal de Santa Catarina, Florianópolis, 2004.

STATGRAPHICS centurion xv. User manual. StatPoint, Inc, 2005. Disponível em: $<$ http://www.statgraphics.com> Acesso em: 22/09/2008.

(Recebido em novembro de 2010. Aceito em maio de 2011.) 